\title{
Remarque générale au sujet du numéro spécial
}

Compte tenu de la nature dynamique de la crise des opioïdes et des données déclarées, ainsi que des différences de définition des mesures, il se peut que les données retrouvées sur les sites Web gouvernementaux et institutionnels soient différentes des données présentées dans ce numéro. 